normal and yet be severely stenosed. The normal passage of meconium and stools is not a reliable guide to the state of the anus, as a stenosed anus will often allow meconium and the soft stool of the newborn to escape. Similarly a rectal thermometer can usually be easily introduced into the rectum in these cases.

The technique of anal examination in the newborn is easy. The anus should first be inspected and then palpated. The little finger, well lubricated, should be used. The finger is inserted into the anus, pad first, very slowly and very gently. The normal anus will stretch as this is done and in most cases a little finger can enter the rectum. However, the examiner's finger may be too big or, as in the case of a very small baby, it may obviously be unwise to over dilate and injure the anus. With practice all degrees of anal stenosis can be excluded without the finger tip entering the rectum. During the palpation two points in particular should be noted. Firstly, the absolute size of the anus. More important than this is the suppleness or otherwise of the canal. A rigid anal canal is an abnormal one.

The anus is easily amenable to examination in the newborn. If there is any doubt that there is even a slight element of stenosis an expert surgical opinion should be urgently obtained.

\section{References}

Partridge, J. P., and Gough, M. H. (1961). Congenital abnormalities of the anus and rectum. British Journal of Surgery, 49, 37-50.

Stephens, F. D., and Smith, E. D. (1971). Anorectal Malformations in Children. Year Book Medical Publishers Inc.: Chicago.

Taylor, I., Duthie, H. L., and Zachary, R. B. (1973). Anal continence following surgery for imperforate anus. Journal of Pediatric Surgery, 8, 497-503.

Correspondence to E. M. Kiely, FRCS, The Children's Hospital, Ladywood Middleway, Ladywood, Birmingham B16 8ET.

\title{
Acute anuric renal failure in an infant with systemic candidiasis
}

\author{
J. Z. HECKMATT, S. R. MEADOW, AND C. K. ANDERSON \\ Department of Paediatrics, University of Leeds, Seacroft Hospital, and the University Department of \\ Pathology, General Infirmary, Leeds
}

SUMMARY We report a newborn baby who presented with acute anuric renal failure resulting from systemic candidiasis. The predisposing factors and diagnostic features are examined.

Although the newborn baby is susceptible to infection and mucocutaneous thrush is common, systemic candidiasis is rare. When it does occur in babies it usually causes osteomyelitis, arthritis, and meningitis. Renal failure is not a recognised feature.

\section{Case history}

A boy was born in August 1977 at 29 weeks' gestation, birthweight $1.430 \mathrm{~kg}$. Respiratory distress syndrome developed and he required ventilation for 23 days. A blood culture taken at $\mathbf{2}$ days grew Staphylococcus aureus and he was given ampicillin and cloxacillin. Subsequently he received courses of gentamicin and carbenicillin.

He was fed intravenously from 3 to 22 days of age via silastic cannulae. These were placed in various veins, including the left long saphenous. At 21 days the long saphenous site became infected and blood cultures grew Candida albicans. The cannula was therefore removed. Five days later $3 \mathrm{ml}$ pus was drained from the abscess; Gram's stain showed yeast cells and mycelial elements; cultures produced a profuse growth of $C$. albicans. He was given benzylpenicillin, streptomycin, and lincomycin from 25 to 30 days and the abscess healed. Thereafter he made good progress and for 5 weeks he fed normally and gained weight. Five blood cultures during this period were sterile.

When he was 64 days old he suddenly became anuric and was transferred to Leeds. There was no urine in the bladder on catheterisation. An intravenous urogram showed nephrograms of 2 normal size kidneys which failed to excrete dye into the renal pelvis. A cystogram was normal; there was no urethral obstruction and no vesico ureteric reflux. After a week of anuria blood tests showed: $\mathrm{Hb}$ $6.4 \mathrm{~g} / \mathrm{dl}, \mathrm{WBC} 14.8 \times 10^{9} / 1\left(14800 / \mathrm{mm}^{3}\right)(75 \%$ 
neutrophils, $24 \%$ lymphocytes), normal platelets, and no evidence of disseminated intravascular coagulation; plasma urea $20 \mathrm{mmol} / 1(120 \mathrm{mg} / 100 \mathrm{ml})$, $\mathrm{K} 6.3 \mathrm{mmol} / 1$.

In order to find out if recovery was likely, and if dialysis was justified, the baby was given an open renal biospy on day 71 . Macroscopically the kidney looked mildly oedematous. The renal pelvis felt normal and contained no obstructing mass. On microscopical examination the renal tissue appeared viable, so peritoneal dialysis was begun the next day and continued for 3 days. Further study of the sections showed Candida within the kidney. Intravenous 5-fluorocytosine therapy was begun with $200 \mathrm{mg} / \mathrm{kg}$ per day initially. Blood levels a thousand times the minimum inhibitory concentration (MIC) of the Candida were obtained (MIC0 $15 \mathrm{mg} / \mathrm{dl}$ ). Two days later he began to pass urine which on microscopical examination contained budding yeast cells and hyphae (Fig. 1).

During this time he also had extensive bronchopneumonia and required further ventilation. The blood culture grew $C$. albicans. There was clinical improvement, the pneumonia resolved and he continued to pass urine. The blood cultures remained positive although the Candida in the blood was

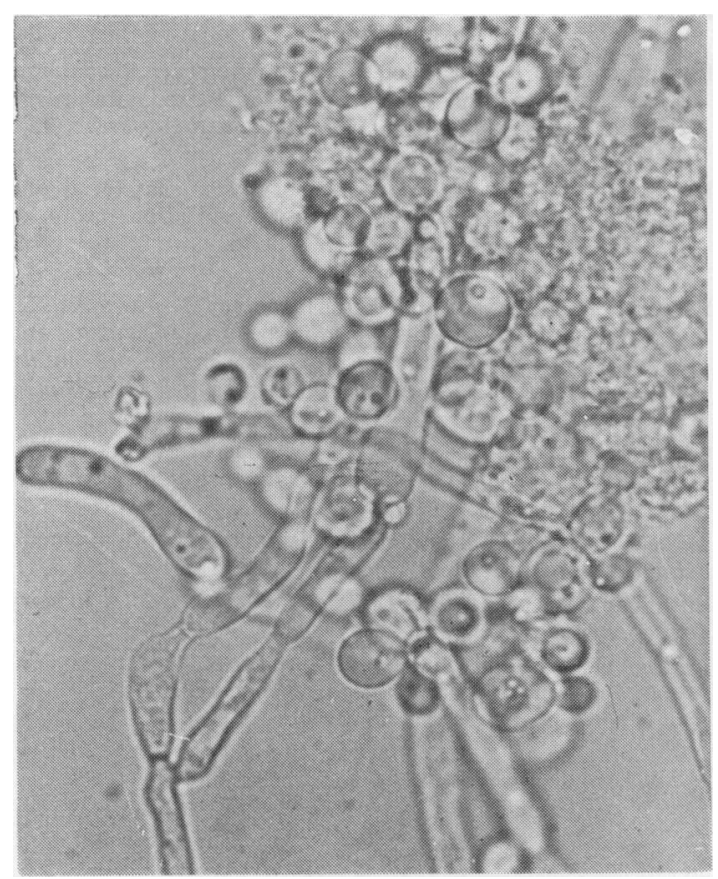

Fig. 1 Unstained urine $H P \times 100$. Budding yeast cells and hyphae. allegedly sensitive to fluorocytosine. For a while clinical improvement was maintained, but he deteriorated suddenly to die at 86 days of septicaemia.

\section{Renal biopsy (Fig. 2)}

A total of 65 glomeruli was seen per section, none being hyalinised or scarred. There was a marked dilatation of Bowman's space in $20 \%$. The tubules were preserved but showed marked epithelial, cloudy swelling, and focal dilatation. At two points there were small foci of necrosis involving tubules and interstitium, accompanied by mixed inflammatory cell infiltration (Fig. 2). Along the side of the biopsy, but not within the areas of necrosis, were abundant fungal filaments which were PAS- and Gram-positive and which stained positively with Grocott's stain for fungi (Fig. 1). These filaments had the morphology of C. albicans. It was considered that the biopsy revealed viable renal tissue which showed toxic and reactive changes, probably due to renal infection with C. albicans.

\section{Necropsy}

The right kidney. This had become a pyonephrosis and was about 4 times normal size. There was a pronounced reduction in renal tissue in some areas and the lower lobe was infarcted.

The left kidney. This was a little enlarged. Small white abscesses were scattered throughout the parenchyma and there were areas of Candida in the interstitium. There was a large abscess, $1 \mathrm{~cm}$ across, occupying the lower lobe.

\section{Discussion}

It is unusual for an apparently healthy baby to develop acute renal failure. The total anuria suggested urinary tract obstruction, but that was excluded by the urogram and cystogram. Intrinsic renal disease was the only alternative. Acute tubular necrosis or renal venous thromboses were unlikely because of the immediate good health of the baby. Despite intensive investigation the cause was not found until Candida was identified in the renal biopsy tissue. It is possible that the anuria was a result of intrarenal obstruction by fungal growth in the renal tubules. Alternatively an 'acute pyelonephritis' shutdown might have occurred from fungal infection.

Systemic candidiasis in infants usually presents as osteomyelitis, arthritis, and meningitis (Businco et al., 1977). In most cases the infant is premature and receiving antibiotics. $A$ vein has usually been catheterised for intravenous feeding (Boeckman and 


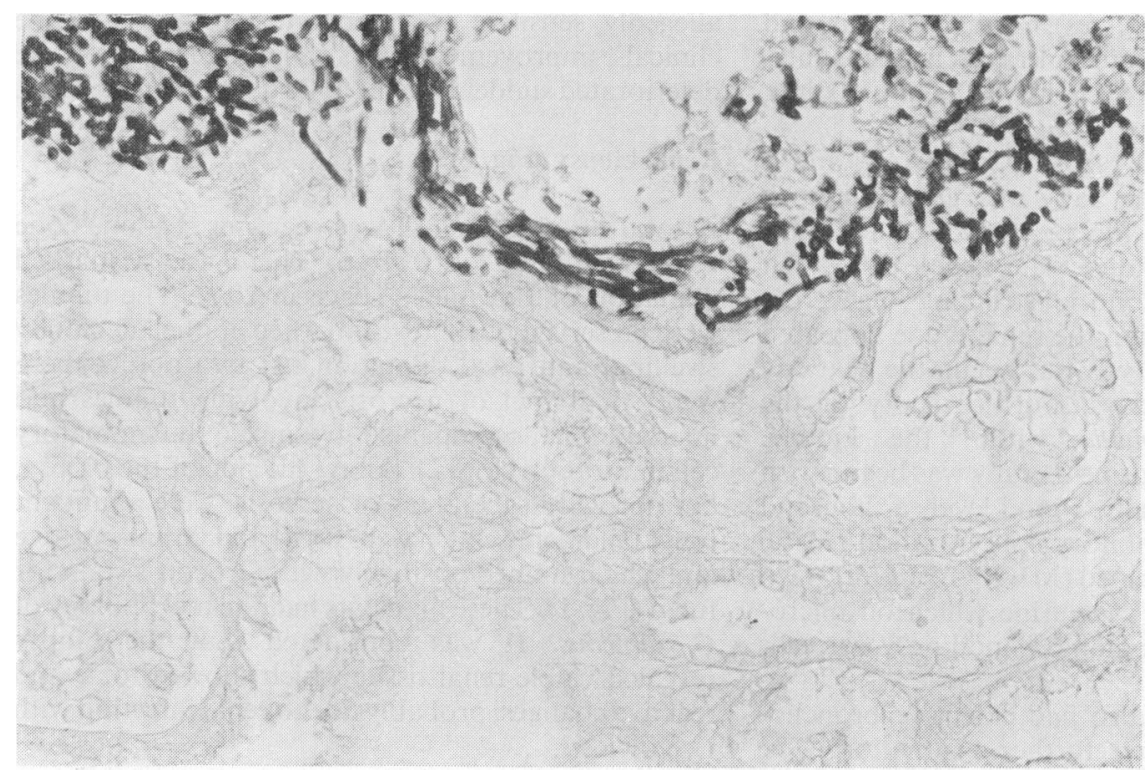

Fig. 2 Renal biopsy (Grocott's stain 285). Positively-staining filaments of Candida albicans are seen at the edge of the renal cortical tissue. Two glomeruli are seen, one on the lower left-hand side, the other on the right.

Krill, 1970). Systemic candidiasis is rare in the healthy infant. However, with the increase in intensive care of the newborn systemic candidiasis may become more common. For effective treatment early diagnosis is essential and there may be clues: the growth of Candida from a cannula tip, or the appearance in the urine of budding yeast cells and, in particular, hyphae (Bernhardt et al., 1974). Indeed urine examination and cultures may be a more reliable method of diagnosis than venous blood culture (Stone, 1974).

Candida infection of the urine, although rare, may occur at any age. Most urine infections are not accompanied by systemic candidiasis or by renal involvement (Littlewood, 1968). Gherardi (1965) reported a baby who presented with severe hypertension resulting from renal artery fungal obstruction. Acute renal failure has not been considered a complication of either systemic or urinary candidiasis in children, although there are reports of its occurrence in adults (Louria et al., 1962; Lehner, 1964).

We thank Dr John Davies for the information about the infant's early life, and Dr Evans and Dr Mason for technical help. We acknowledge, with gratitude, the sustained efforts of the nursing and medical staff in Grimsby and Leeds; their help so nearly succeeded in saving the baby.

\section{References}

Bernhardt, H. E., Orlando, J. C., Benfield, J. R., Hirose, F. M., and Foos, R. Y. (1974). Disseminated candidiasis in surgical patients. Surgery, Gynecology, and Obstetrics, 134, 819-825.

Boeckman, C. R., and Krill, C. E. (1970). Bacterial and fungal infections complicating parenteral alimentation in infants and children. Journal of Pediatric Surgery, 5, 117-126.

Businco, L., Iannaccone, G., Del Principe, D., Lucarelli, S., Cardi, E., and Rezza, E. (1977). Disseminated arthritis and osteitis by Candida albicans in a two-month-old infant receiving parenteral nutrition. Acta paediatrica Scandinavica, 66, 393-395.

Gherardi, G. J., (1965) Systemic moniliasis in infancy: fatal renal hypertensive complications. Journal of the American Medical Association, 193, 155-157.

Lehner, T. (1964). Systemic candidiasis and renal involvement. Lancet, 1, 1414-1416.

Littlewood, J. M. (1968). Candida infection of the urinary tract. British Journal of Urology, 40, 293-305.

Louria, D. B., Stiff, D. P., and Bennett, B. (1962). Disseminated moniliasis in the adult. Medicine, 41, 307-337.

Stone, H. H. (1974). Studies in the pathogenesis, diagnosis, and treatment of Candida sepsis in children. Journal of Pediatric Surgery, 9, 127-133.

Correspondence to Dr J. Z. Heckmatt, Department of Paediatrics and Child Health, Medical Education Centre. Seacroft Hospital, York Road, Leeds LS14 6UH. 